\title{
What and How Much to Gain by Spectrum Agility?
}

\author{
Chun-Ting Chou, Sai Shankar N, Hyoil Kim, and Kang G. Shin
}

\begin{abstract}
Static spectrum allocation prohibits radio devices from using spectral bands designated for others. As a result, some bands are under-utilized while other bands are over-populated with radio devices. To remedy this problem, the concept of spectrum agility has been considered so as to enable devices to opportunistically utilize others' spectral bands. In order to help realize this concept, we establish an analytical model to derive performance metrics, including spectrum utilization and spectrum-access blocking time in spectral-agile communication systems. We then propose three basic building blocks for spectralagile systems, namely spectrum opportunity discovery, spectrum opportunity management, and spectrum usage coordination, and develop protocols for each blocks. These protocols are integrated with the IEEE 802.11 protocol, and simulated using $n s-2$ to evaluate the protocol overhead. The simulation results show that our proposed protocols can improve the throughput of an IEEE 802.11 wireless LAN by $90 \%$ for the simulated scenarios, and the improvements matched well our analytical model. These results demonstrate the great potential of using spectrum agility for improving spectral utilization in an efficient, distributed, and autonomous manner.
\end{abstract}

Index Terms-spectrum agility, cognitive radios, programmable wireless networks

\section{INTRODUCTION}

$\mathbf{C}$ ONVENTIONAL wireless devices are only allowed to operate in designated spectrum bands due to regulatory restrictions. Within each designated band, radio devices adopt specific communication protocols and use fixed modulation and medium access control schemes. Even though such designs simplify protocol and hardware development, there exists one major potential problem with them, namely, inefficient utilization of precious spectral resources. The main cause of this inefficiency is that radio devices in crowded spectral bands are prohibited from (due to regulations), and incapable of (due to hardware limitation), using other idle or less crowded spectrum bands. Such spectrum inefficiency is becoming a serious problem as more and more communication protocols and wireless technologies are being developed to operate in crowded unlicensed spectrum bands.

Recently, spectral-agile or cognitive radios have drawn considerable attention for their potential to alleviate the spectrum

Manuscript received February 1, 2006; revised November 1, 2006. The work reported in this paper was supported in part by the NSF under Grant No. CNS 0519498.

Chung-Ting Chou is with Philips Research North America, 345 Scarborough Road, Briarcliff Manor, NY 10510, USA (e-mail: chunting.chou@philips.com).

Sai Shankar N is with Corp. R\&D Systems Engineering department at Qualcomm Inc., 5775 Morehouse Drive, San Diego, USA (e-mail: sai.shankar@qualcomm.com). This work was done when he was with Philips Research North America.

Hyoil Kim and Kang G. Shin are with Real-Time Computing Laboratory, The University of Michigan, Ann Arbor, MI 48109-2122, USA (e-mail: \{hyoilkim,kgshin\}@umich.edu).

Digital Object Identifier 10.1109/JSAC.2007.070408. scarcity problem. For example, the US Federal Communications Commission (FCC) has issued a Notice of Public Rulemaking and Order regarding cognitive radio technologies [1]. The Defense Advanced Research Projects Agency (DARPA) has started the neXt Generation (XG) Communications Program to develop new technologies which allow multiple users to share the spectrum through adaptive mechanisms [2]. The US Army has also been researching "Adaptive Spectrum Exploitation" (ASE) for real-time spectrum management in the battlefield [3][4]. New standardization activities, such as IEEE 802.22, have also targeted the use of TV bands for spectral-agile wireless regional access networks (WRANs) [5]. Although these programs have somewhat different focuses, their basic principles are the same: if radio devices are allowed to explore the wireless spectrum, they may be able to discover unused spectral bands and exploit them opportunistically. By doing so, not only the devices' performance but also the overall spectrum utilization can be improved. In the long run, this may lead to secondary markets for unused licensed bands (i.e., a licensee may allow his spectrum to be used by a third party) or automated coordination of frequency allocation among different radio systems [1].

The benefits of using spectrum agility cannot be achieved without developing new hardware/software and changing the current spectrum allocation policy. Fortunately, the advances in software defined radio (SDR) [6][7] have enabled the development of flexible and powerful radio interfaces for supporting spectral agility. The FCC's ongoing review of the current spectrum regulations is also expediting the adoption of more flexible spectrum policies. However, there remain many open questions that must be answered with respect to spectralagile communications.

The first and the foremost question to answer is how much improvement in terms of spectrum utilization one can gain by using spectrum agility. Without a clear answer to this fundamental question, one can justify neither the use of spectrum agility nor the investment into this technology. Other key issues include how to discover and identify unused spectral bands, and when to utilize them by which mechanisms or protocols. Obviously, different protocols incur different amounts of control overhead, which, in turn, result in different degrees of performance degradation.

In this paper, we address these issues mostly at the medium access control (MAC) layer and its related aspects. We first define a reference model for generic spectral-agile communications to facilitate protocol development. We then establish an analytical model to derive performance bounds, including spectrum utilization and spectrum-access blocking time, in a spectral-agile system. The analysis will be used as a performance benchmark to evaluate our protocols/implementations. 
Based on our reference model, a set of new protocols are developed to make an IEEE 802.11 wireless LANs spectrally agile. These protocols are integrated with the IEEE 802.11 standard and implemented in $n s-2$. By using our analytical model and simulations, we show the advantages of using spectrum agility and the effectiveness of our protocol designs.

It should be noted that spectrum agility addressed in this paper differs significantly from the problems of so-called dynamic frequency-selection, such as Dynamic Channel Selection [9] in cellular networks, Dynamic Frequency Selection [10] in the IEEE 802.11h standard or Auto Frequency Allocation [11] in the HiperLAN. These schemes address the problem of choosing a channel from a pre-defined spectral band to mitigate interference between devices. However, a spectral-agile device faces the problems of (1) seeking spectral resources outside its allocated bands, (2) coexistence and resource-use coordination with both peer and incumbent devices, and (3) using spectral resources opportunistically. In fact, dynamic frequency selection (DFS) is a subset of spectral-agile communications, and therefore, the models and solutions developed here can be applied to DFS as well.

The rest of this paper is organized as follows. The system model and assumptions are introduced in Section II. The analytical model and some numerical results are presented in Section III. In Section V, the details of the spectralagility-related functionalities are presented, and in Section VI the simulation results are analyzed and discussed. Finally, conclusions are drawn in Section VII.

\section{System Model}

We consider two types of devices: primary and secondary devices. Primary devices are referred to as those that have exclusive access to some parts of the spectrum. All current licensed-band devices — such as TVs, cellular phones or radars/satellites - are therefore considered as primary devices in this paper. Secondary devices are referred to those without pre-allocated spectral bands and may need to share spectral resources with other secondary devices. Unlike conventional unlicensed-band devices in $900 \mathrm{MHz}$, Industrial Scientific Medical (ISM) bands in $2.4 \mathrm{GHz}$ or UNI II and UNI III bands at $5 \mathrm{GHz}$, we assume that secondary devices are spectrally agile, and are thus capable of seeking and opportunistically utilizing spectral resources in both licensed and unlicensed bands.

Due to hardware limitation such as antennas or RF frontends, secondary devices may not be able to access the entire wireless spectrum. Therefore, the term "wireless spectrum" in this paper is referred to as the portion of the spectrum accessible to secondary devices. The spectrum is further divided into "channels", each representing a fixed, minimum amount of bandwidth. For example, one can imagine a spectral-agile IEEE 802.11-type device to access the spectrum of $2.4 \mathrm{GHz}$ to $5 \mathrm{GHz}$ where there are a total of $13020-\mathrm{MHz}$ channels. We assume that each secondary device only requires a single channel for transmission. However, a secondary device may be able to use multiple channels simultaneously. Such a device is usually equipped with either multiple transceivers with narrow-band antennae or a single wideband transceiver. For

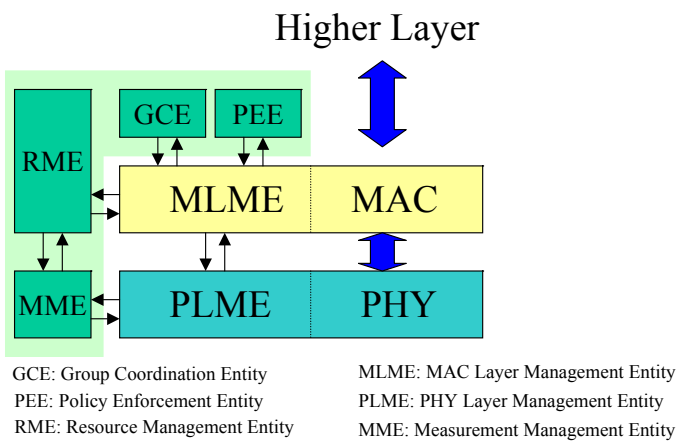

Fig. 1. Reference model of spectral-agile secondary devices.

example, an Ultra-wideband (UWB) device using Orthogonal Frequency Division Multiplexing (OFDM) can switch on and off the corresponding subcarriers based on channel availability, and can thus access multiple idle channels simultaneously.

Figure 1 shows the system architecture of a typical spectralagile secondary device. As shown in the figure, each device has four new control entities, including (1) Policy Enforcement Entity (PEE), (2) Measurement Management Entity (MME), (3) Resource Management Entity (RME), and (4) Group Coordination Entity (GCE). In general, these entities use medium access control (MAC)- and physical (PHY)layer functionalities via the MAC-Layer Management Entity (MLME) and PHY-Layer Management Entity (PLME) to perform their tasks.

As suggested by its name, PEE ensures that secondary devices comply with regulatory requirements when accessing the licensed bands. In this paper, we only consider one simplified requirement; that is, secondary devices use a channel if and only if they do not interfere with the primary devices of that channel. In other words, secondary devices must cease all transmissions on a channel upon return of the primary devices. The actual regulatory requirements are far more complicated and the details are beyond the scope of this paper. Therefore, we will focus only on MME, RME and GCE. The key functions of these three control entities are summarized as follows.

- MME performs in-band and out-of-band channel sensing to monitor spectral activities. MME may initiate channel sensing periodically or upon request from RME. We assume that all spectral-agile devices use the same MAC and PHY protocols and therefore, can detect each other's signals easily. To detect signals of primary devices, the secondary devices need to use so-called feature detection techniques. Feature detection itself is a very challenging research problem, especially when the received signal strength is under noise levels or thresholds that are far below the sensitivity levels of primary devices [15]. Fortunately, many random signals encountered in the field of wireless communications are cyclostationary because of underlying periodicity due to various operations such as sampling, scanning and modulation. If a signal exhibits strong cyclostationary properties, it can be detected at very low signal-to-noise ratios (SNR) 
and separated from noise, which is typically stationary as opposed to cyclostationary [16][17]. Throughput out this paper, we assume that secondary devices rely on cyclostationary detectors to detect the presence of primary devices. Therefore, our discussion about MME focuses on the algorithms/protocols that control the sensing, instead of PHY-layer sensing itself.

- RME keeps track of spectral activities in each channel and identifies usable spectral resources. RME obtains information of spectral activities through periodic updates from MME and updates sent by other secondary devices, or by initiating on-demand sensing requests. Whenever a currently-occupied channel becomes unavailable (e.g., reclaimed by its primary devices) or its condition degrades, RME selects another usable channels and requests the GCE to coordinate other communicating peers to switch to the chosen channel.

- GCE coordinates the channel selection within a communication group or between different communication groups. A communication group is referred to as a group of secondary devices that communicate with each other. For example, an IEEE 802.11 access point with the associated wireless stations is considered as a communication group. GCE ensures that all devices in the same communication group use the same channel (since the RME of each device may select a different channel). Such coordination is called intra-group coordination, and is achieved via an in-band signaling using the same MAC protocol for data transmission. GCE also ensures that different communication groups use different channels, if possible, to maximize overall spectrum utilization. Such coordination is called inter-group coordination, and is achieved via a pre-defined etiquette. We assume that devices between different communication groups do not exchange information for security reasons.

In the next section, we will establish a simple mathematical model to analyze the performance of ideal spectral-agile devices. That is, the MME, RME and GCE perform perfectly without incurring any control overhead. This model will provide a performance benchmark and will be used to evaluate our protocols for spectral-agile communication systems in Section VI.

\section{Analytical Model for Ideal Spectral-Agile DEVICES}

The temporal usage of each channel, ${ }^{1}$ by the primary devices, is modeled as a random process. Since primary devices do not always use their designated channels, some channels may be left unused for certain periods of time during which these channels may be exploited by spectral-agile secondary devices. These idle intervals, referred to as spectral opportunities, vary with primary devices' usage patterns. For example,

\footnotetext{
${ }^{1}$ A channel could be a frequency band in Frequency Division Multiple Access (FDMA) systems, a set of time slots in Time Division Multiple Access (TDMA) systems, or a spreading code in Code Division Multiple Access systems
}

the opportunities can last up to several hours or even days in emergency police bands, but last for only few milliseconds in heavily-used spectral bands. For the long-lasting opportunities, it is relatively easy for spectral-agile devices to discover and utilize them. However, for the very short-lasting opportunities, spectral-agile devices may never detect the existence of these opportunities, not to mention to utilize them. Therefore, we only focus on the practical cases where spectral opportunities last in the order of seconds or longer.

We consider a system where there are $\mathrm{N}$ channels available for spectral-agile radio devices. The usage pattern of primary devices, in each channel, is assumed to follow i.i.d. ON/OFF random process. An ON-period represents that the channel is being used by primary devices and thus is unavailable to spectral-agile devices. An OFF-period represents that the channel can be used by spectral-agile devices and thus is considered as a spectral opportunity. The ON- and OFFperiods on each channel are assumed to be independent and the mean values of the ON and OFF periods are dictated by primary devices' usage patterns. To simplify our analysis, we assume that the distributions of both ON- and OFF-periods on each channel are exponentially-distributed with means equal to $T_{o n}$ and $T_{o f f}$, respectively. We will explore different distributions using simulation at the end of this section.

We assume that there are $M$ spectral-agile communication groups seeking spectral opportunities in the $N$ channels. Note that a "group", instead of a device, is considered as the smallest entity to exploit spectral opportunities. For example, a spectral-agile wireless LAN composed of one access point and two devices are considered as one group in our analysis. This is mainly because all 3 devices have to use the same channel all the time in order to maintain connectivity. Once the group acquires an opportunity via GCE, individual devices will share the opportunity via the underlying MAC. Each group is assumed to have an infinite amount of traffic to transmit.

We consider two performance metrics, spectrum utilization and spectrum-access blocking time. The spectrum utilization is defined as the percentage of time that a spectral-agile group has some channels for transmission. If multiple groups discover the same opportunity and share it with each other, the amount of time contributed to each group's spectrum utilization is evenly divided. Based on the spectrum utilization, we can calculate the throughput once the underlying MAC and modulation mechanisms are specified. Therefore, our analysis is not confined to any specific MAC protocols or modulation schemes. The spectrum-access blocking time is defined as the duration when a spectral-agile group finds no spectral opportunity to transmit (not including the waiting time when sharing with other spectral-agile groups).

In order to evaluate the improvement, we use the performance of non-agile secondary groups for comparison. A nonagile group can only access a fixed channel when the channel is not used by its primary devices. The spectrum utilization of such a non-agile group can be easily computed as

$$
U_{\text {nonagile }}=\frac{T_{o f f}}{T_{o n}+T_{o f f}},
$$

and the average spectrum-access blocking time is $T_{o n}$. In what 
follows, we will derive the spectrum utilization of spectralagile groups and compare it with $U_{\text {nonagile }}$ given in Eq. (1).

\section{A. A Special Case: Only one secondary group $(M=1)$}

For simplicity, let us first consider a special case where there is only one spectral-agile group. As shown in Figure 2, the only time duration when this group has no channel for traffic transmission is when all channels are occupied by the primary devices. Such blocking intervals, denoted as $t_{\text {block}}$, always begin when a channel switches from an OFF-period to an ON-period and ends when any one channel switches from an ON-period to an OFF-period. Therefore, $t_{\text {block }}$ can be computed as

$$
t_{\text {block }}=\min _{i=1,2, \cdots, N}\left(T_{\text {remain }}^{(i)}\right),
$$

where $T_{\text {remain }}^{(i)}$ is the remaining ON-period (i.e., residual occupancy time of the primary devices) on channel $i$. Since the ON-periods are independent and exponentially distributed, one can compute the distribution of $t_{\text {block }}$ as

$$
P\left(t_{\text {block }}=t\right)=\frac{N \cdot e^{-\frac{T_{o n}}{N} t}}{T_{\text {on }}} .
$$

Eq. (3) shows that the average spectrum-access blocking time of a spectral-agile group is reduced to $\frac{T_{o n}}{N}$, compared to $T_{o n}$ of a non-agile group. The spectrum utilization of a spectral-agile group is obtained by

$$
U_{\text {agile }}=1-\frac{N\left(p^{N-1} \cdot \frac{T_{o n}}{N}\right)}{T_{o n}+T_{o f f}}
$$

where $p=\frac{T_{o n}}{T_{o n}+T_{o f f}}$ is the probability that a channel is occupied by the primary devices. Eq. (4) is derived from the fact that a blocking interval starts only if a channel switches from an OFF-period to an ON-period while all other channels have already been in their ON-periods. It should be noted that $p$ has the same expression even when the ON- and OFFperiods are generally distributed. Eq. (4) can be simplified further to

$$
U_{\text {agile }}=1-\left(\frac{T_{\text {on }}}{T_{\text {on }}+T_{\text {off }}}\right)^{N},
$$

showing that the spectrum utilization of a spectral-agile group is a simple function of the primary devices' channel utilization. We can generalize this simple equation for the case when different channels have different distributions of $\mathrm{ON}$ - and OFF-periods. Let channel $i$ have the mean ON- and OFFperiods indicated by $T_{o n}^{i}$ and $T_{o f f}^{i}$, respectively. Then, the utilization of channel $i$ is equal to $\tau_{i}=\frac{T_{o n}^{(i)}}{T_{o n}^{(i)}+T_{o f f}^{(i)}}$. Eq. (5) can then be rewritten for the general case as

$$
U_{\text {agile }}=1-\prod_{i=1}^{N} \tau_{i}
$$

Finally, the improvement of the spectrum utilization achieved by a spectral-agile group is computed as

$$
I=\left(\frac{U_{\text {agile }}}{U_{\text {nonagile }}}-1\right) * 100 \%,
$$

where $U_{\text {nonagile }}$ is given by Eq. (1).

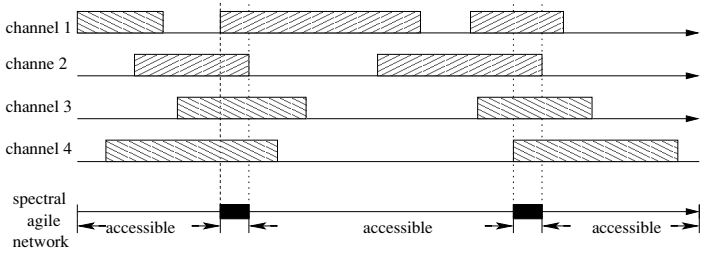

Fig. 2. A special case: $\mathrm{N}=4$.

\section{B. The General Case: Multiple spectral-agile group $(M>1)$}

Eq. (5) shows that the spectrum utilization of a spectralagile group is simply a function of the primary devices' channel utilization, $\tau=\frac{T_{o n}}{T_{o n}+T_{o f f}}$. We will now consider the case when there are more than one spectral-agile group $(M>1)$ that are trying to opportunistically access the available channels. First, we have to compute the number of available channels (that are not occupied by the primary devices) and then "distribute" them among those spectral-agile groups ${ }^{2}$. Based on Eq. (6), the fraction of time during which there are $k$ channels available simultaneously is computed as

$$
r_{k}=\sum_{c=1}^{\frac{N !}{k !(N-k) !}}\left[\prod_{i \in S_{c}^{k}}\left(1-\tau_{i}\right) \prod_{j \in\{1,2, \cdots, N\}-S_{c}^{k}} \tau_{j}\right],
$$

where $S_{c}^{k}$ is a set of $k$ channels, chosen from $N$ channels, which are available for spectral-agile groups. For example, we can set $S_{1}^{k}=\{1,2, \cdots, k\}, S_{2}^{k}=\{2,3, \cdots, k+1\}$, and so on.

If there are more than $M$ channels available simultaneously, each group will obtain exactly one channel. Otherwise $(k<$ $M)$, the $M$ groups have to share $k$ channels via the GCE. By combining these two cases, the spectrum utilization of each spectral-agile group is then computed by

$$
U_{\text {agile }}=\sum_{k=0}^{N} \frac{\min (M, k) r_{k}}{M} .
$$

Given that there are $M>1$ groups competing for spectral resources, $U_{\text {nonagile }}$ in Eq. (1) must be derived differently. We consider two straightforward cases: (1) each non-agile group randomly selects its own channel independently of others, and (2) all non-agile groups choose different channels, if possible, possibly via an off-line channel allocation policy. Note that once the channel is chosen, each group will not dynamically switch between channels to seek opportunities as spectral-agile groups do. We derive $U_{\text {nonagile }}$ for these two cases in the following subsections.

1) Random Channel Selection: To derive $U_{\text {nonagile, we }}$ need to determine the probability that some non-agile groups choose the same channel. Given that a non-agile group chooses channel $i$, the probability that the other $h$ groups also choose the same channel is

$$
p_{h}=\frac{(M-1) !}{h !(M-1-h) !}\left(\frac{1}{N}\right)^{h}\left(\frac{N-1}{N}\right)^{M-1-h} .
$$

\footnotetext{
${ }^{2}$ We assume in this section that GCE works perfectly. Therefore, different groups use different idle channels, if possible, via inter-group coordination, and thus maximize their spectrum utilizations
} 

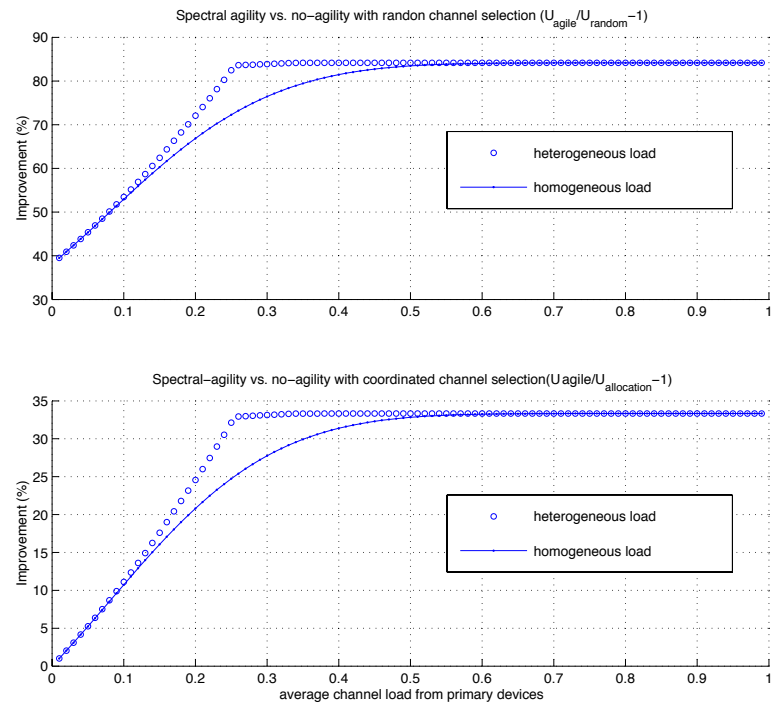

Fig. 3. Improvement of spectral utilization for spectral-agile groups: $N=$ 12 and $M=9$. *Although the figure shows the maximal improvement percentage $(82 \%)$ occurs when the channel load approaches 1 , it does not suggest that spectrum agility generates the greatest amount of spectral opportunities. Instead, it shows that, for example, with load of 0.99 , the average channel access time for a spectral-agile node increases from $0.01=1-0.99$ (i.e., no-agility) to $0.0182 \mathrm{sec}$ out of a 1 -second period as also shown in Figure 4.

Therefore, the fraction of time a non-agile group can transmit, given that it has chosen channel $i$, is

$$
T_{i}=\sum_{h=0}^{M-1} p_{h} \frac{T_{o f f}^{(i)}}{(h+1)\left(T_{o n}^{(i)}+T_{o f f}^{(i)}\right)} .
$$

Here, we assume that the groups that choose the same channel fairly share the spectrum resource via the underlying MAC protocol. The spectrum utilization $\left(U_{\text {nonagile }}\right)$ of each nonagile group can then be computed as

$$
U_{\text {random }}=\frac{1}{N} \sum_{i=1}^{N} T_{i} .
$$

The improvement obtained by spectral-agile groups over nonagile groups is then given by

$$
I_{\text {random }}=\left(\frac{U_{\text {agile }}}{U_{\text {random }}}-1\right) * 100 \% .
$$

2) Off-line Channel Allocation: In this case, each nonagile group is given a channel for transmission whenever the channel becomes idle. The fraction of time each non-agile group can obtain is computed as

$$
U_{\text {allocation }}=\frac{\sum_{c=1}^{\frac{N !}{M !(N-M) !}} \frac{1}{M} \sum_{i \in S_{c}^{M}} \frac{T_{o f f}^{(i)}}{T_{o n}^{(i)}+T_{o f f}^{(i)}}}{\frac{N !}{M !(N-M) !}} .
$$

Here, we simply average all the possibilities of choosing $M$ channels from $N$ channels. We set $\frac{N !}{M !(N-M) !}=1$ in case of $M>N$. The improvement obtained by spectral-agile groups over non-agile groups is then given by

$$
I_{\text {allocation }}=\left(\frac{U_{\text {agile }}}{U_{\text {allocation }}}-1\right) * 100 \% .
$$

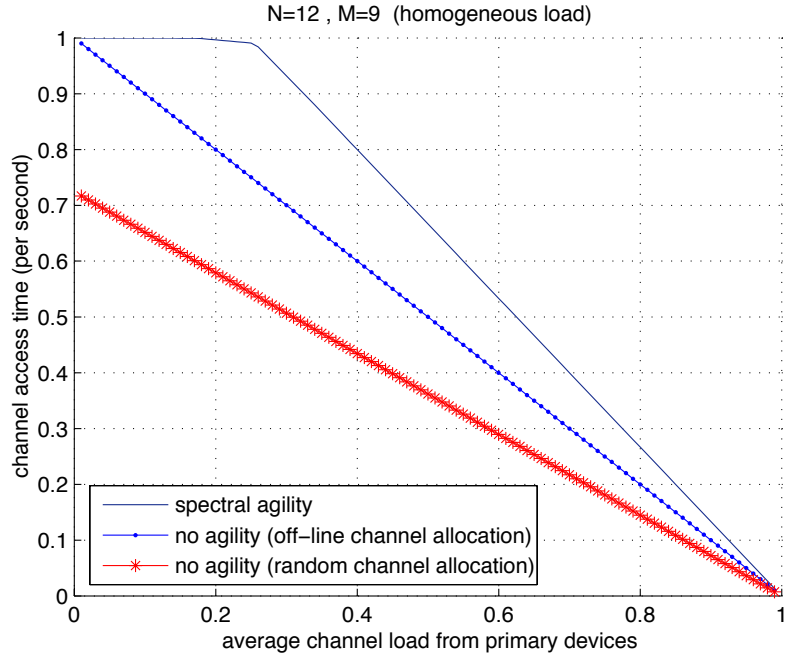

Fig. 4. Fraction of channel access time: $N=12$ and $M=9 .{ }^{*}$ This figure, together with Figure 3, suggest that a spectral-agile secondary group benefits most from spectral agility when the channel utilization of primary devices is lightly$(0.2)$ or moderately-loaded $(0.7 \sim 0.8)$.

\section{NUMERICAL ANALYSIS}

Having derived the spectrum utilization of spectral agile devices, we would now like to compare the performance of spectral-agile devices with (1) non-agile devices with random channel selection, and (2) non-agile devices with off-line channel allocation, based on Eqs. (9), (12), and (14) in the IEEE $802.11 \mathrm{a} / \mathrm{b} / \mathrm{g}$ ISM and UNI bands. We investigate two scenarios with $N=12$ and $N=3$, respectively. The main reason for choosing these numbers is that there are 12 (nonoverlapping) channels in the 5-GHz band for the IEEE 802.11a wireless LAN and 3 (non-overlapping) channels in the 2.4$\mathrm{GHz}$ band for the IEEE $802.11 \mathrm{~b}$ wireless LAN. Note that this comparison is done to highlight the advantages of spectral agility (it is easy to derive other examples using cellular or other bands). Since IEEE 802.11 has proliferated the home and enterprise markets to a great extent and operates using a listenbefore-talk protocol, it will be one of the prime candidates wherein the industry would choose to make it spectral agile. Even though spectrum agility cannot be applied immediately to the licensed bands due to the current regulations, the 802.11 wireless LAN may use spectrum agility to improve performance in the crowded, unlicensed bands.

3) Case 1: $(M<=N)$ : Figure 3 shows the case of $N=12$ and $M=9$ with different average channel loads generated by the primary devices. The loads in these 12 channels can be either homogeneous or heterogeneous. In case of homogeneous loads, each channel is assigned a load equal to the average channel load, while, in case of heterogeneous loads, different channels are assigned different loads which maximize the load variance (i.e., the utilization of each channel differs significantly from each other). The improvement shown in Figure 3 is defined as

$$
\text { improvement }(\%)=\left(\frac{U_{\text {agile }}}{U_{\text {random } / \text { allocation }}}-1\right) \cdot 100 \% \text {, }
$$

where $U_{\text {agile }}, U_{\text {random }}$, and $U_{\text {allocation }}$ are given in Eqs. (9), (12), and (14), respectively. The results show that use of spectrum agility always achieves a higher spectrum utilization 


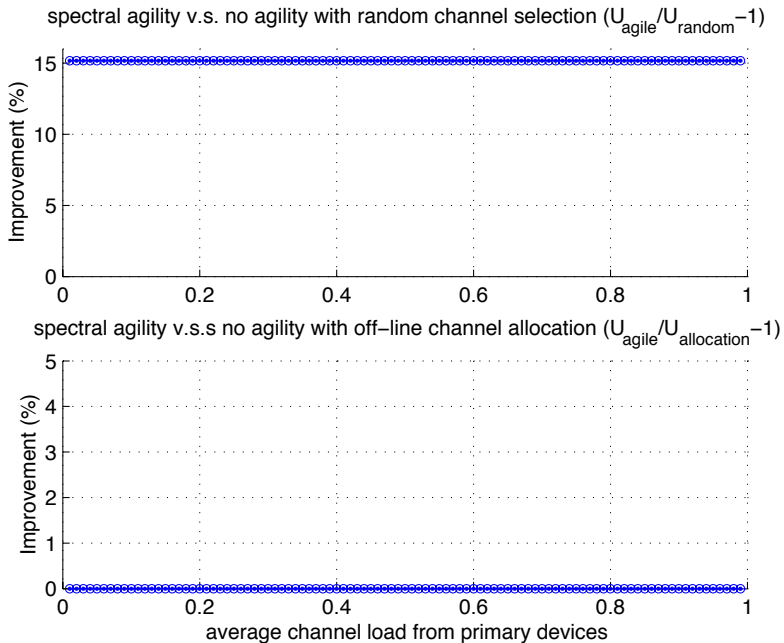

Fig. 5. Improvement of spectral utilization for spectral-agile devices: $N=3$ and $M=5$. *The figures show that when the number of available channels is less than the number of secondary devices, off-line channel allocation. However, spectrum agility still outperforms non-agile random channel selection.

for a secondary group than the case of no agility with random channel selection or off-line channel allocation. Of course, the improvement by using spectral agility is smaller (still more than $25 \%$ in most cases) when compared to the case of no agility with off-line channel allocation (Figure 3(b)). However, the off-line channel allocation needs a priori channel information. If the channel loads range widely, using spectrum agility allows a secondary group to dynamically choose the channel with the least activities. Such advantages are also illustrated in Figure 3, where we achieve an extra 8$10 \%$ improvement for heterogeneous loads when the channel load is around $0.2 \sim 0.3$.

An interesting observation is that the improvement ratio (i.e., Eq. (16)) saturates when the channel load of the primary devices is greater than 0.5. This can be explained by Figure 4, which shows that the fraction of time (for which a secondary group can access a channel) decreases linearly with the increase of the average channel load in all three cases when the channel load exceeds 0.3. Because of such linearity, the improvement ratio of using spectrum agility, as compared to no-agility cases, remains unchanged when the channel load is greater than 0.3 as shown in Figure 3. Figure 4 also suggests that when the average channel load generated by the primary devices is very large, it does not make much sense to use spectral agility as indicated by Figure 3 (even though it shows an $80 \%$ improvement with the load of 0.9 ). This is because when the channel is highly loaded, the amount of accessing time that each spectral-agile device can acquire is very small (less than $10 \%$ of the total time with the channel load of 0.9). Therefore, the control overhead (which is not considered here) may use up most of the channel access time a secondary device acquires, hence easily offsetting the improvement made by spectrum agility.

4) Case II: $(M>N)$ : Next, we consider the case of $M>$ $N$ and choose $N=3$ and $M=5$ as an example. Figure 5-(b) shows that using spectrum agility and using no agility with off-line channel selection yield exactly the same performance (i.e., no improvement). This is because when $M>N$, there
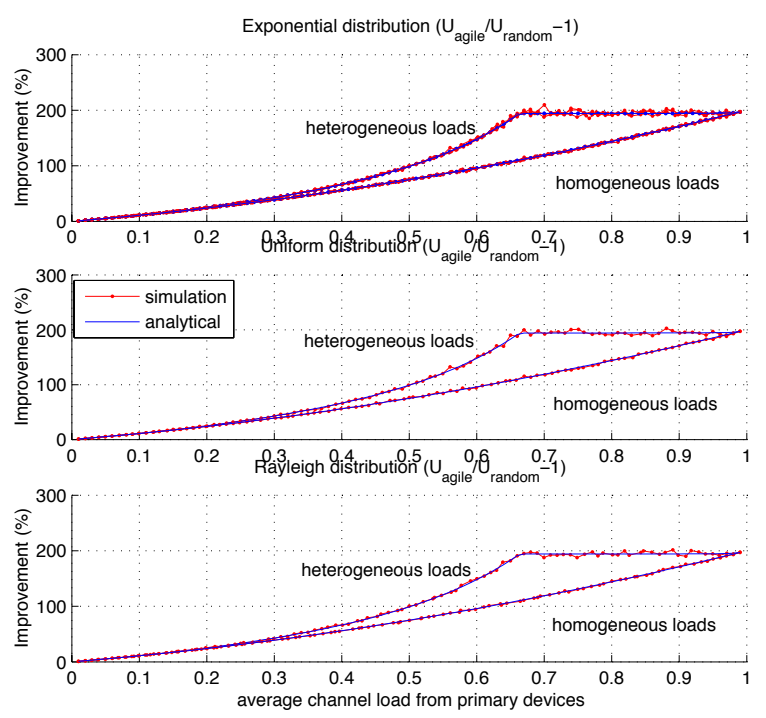

Fig. 6. Improvement of spectral utilization for spectral-agile devices: different ON/OFF distributions *Although the figure shows the maximal improvement percentage $(200 \%)$ occurs when the channel load approaches 1 , it does not suggest that spectral agility generates the greatest spectral opportunities. Instead, it shows that, for example, with load of 0.99 , the average channel access time for a spectral-agile node increases from $0.01=1-0.99$ (i.e., no-agility) to $0.03 \mathrm{sec}$ out of a 1 -second period, similar to what shows in Figure 3.

are simply not enough channels for all secondary devices (so they have to share idle channels with each other). In fact, one can simplify both Eqs. (9) and (14) as

$$
U_{\text {agile }}=U_{\text {coordinated }}=\frac{1}{M} \sum_{i=1}^{N} \frac{T_{o f f}^{(i)}}{T_{o n}^{(i)}+T_{o f f}^{(i)}}
$$

when $M>N$, which verifies the result in Figure 5-(b). There are some marginal improvements by using spectrum agility as compared to using no agility with random channel selection as shown in Figure 5-(a). This is simply because some idle channels may be left unused in the case of random channel selection.

Figures 3 and 5 show that radio devices can benefit from spectrum agility only when there are enough spectral resources to be exploited (i.e., $M<N$ ). Fortunately, field studies have shown that there are many under-utilized spectral resources in many wireless spectral bands [12][13]. Moreover, there are two additional advantages of using spectrum agility that we have not yet discussed when $M>N$. First, Eq. (3) shows that when the spectrum agility is used, the average blocking time is reduced by a factor of $N$ in the special case or reduced from $\frac{\sum T_{o n}^{(i)}}{N}$ to $\frac{1}{\sum \frac{1}{T_{o n}^{(i)}}}$ in the general case. Thus, even though the spectral utilization is not improved by using spectrum agility when $M>N$, the packet delays are reduced significantly by using spectrum agility. Another advantage is the spectral-agile device's capability of using multiple channels. In the above analysis, we assumed that a spectral-agile device uses only one channel, even when more than one channel is available. We can expect that if a spectral-agile device can use all available channels, the performance can be further improved. 


\section{A. Effect of different ON/OFF distributions on spectral uti- lization}

We now investigate the effects of different ON/OFF distributions on the improvement of spectral utilization by using spectrum agility. The main purpose of this study is to verify the applicability of our model, which is established based on the assumption of exponentially-distributed ON-/OFF periods. Here, we use Matlab to simulate the random ON/OFF periods and calculate the total time intervals of overlapping ONperiods (i.e., the blocking intervals for a spectral-agile device) for the case of $N=3$ and $M=1$. We use exponential (as in our earlier derivation), uniform, and Rayleigh distributions to model the ON- and OFF-periods of primary devices. Figure 6 shows a very good match between our analytical results and the simple simulation results, demonstrating the applicability of our analytical model. The reason why the improvement ratios (again as defined in Eq. (16)) are much higher (up to $200 \%$ ) is that there is only one spectral-agile group seeking spectral opportunities, and thus, it need not share spectral opportunities with other spectral-agile groups. However, as discussed earlier, such a large improvement ratio, in fact, represents only a very small increase of actual channel access time for a secondary group when the channel load generated by the primary device is extremely high. Therefore, one should not expect improvement, given the control overhead incurred by spectral agility, when the average channel load of the primary device is very high. This will be shown in Section VI using our $n s$-2-based simulation.

\section{SPectral-agility Functionalities}

As mentioned in Section II, a spectral-agile device relies on MME, RME and GCE for spectral opportunity discovery, opportunity management, and coordination of opportunity uses, respectively. We now detail the general protocols and algorithms used by these three control entities. These protocols and algorithms are then tailored to the IEEE 802.11 standard in Section VI in order to realize a spectral-agile IEEE 802.11 wireless LAN.

\section{A. Measurement Management Entity (MME)}

The MME of each device executes periodic scanning to discover spectral opportunities. Upon each scheduled scan, the MME chooses a channel from those that are neither the currently-occupied channel nor the channel chosen for the previous scan. The MME may select such a channel either in a random or round-robin manner. However, if the device has detected primary devices in the currently-occupied channel, the scheduled scan is canceled. This is because if the device switches to the chosen channel for scanning, it may lose communication with other devices, thus not being able to coordinate with each other via their GCEs to switch to another idle channel (details are discussed in the next subsections). While a scheduled scan is being executed, the device stays in the SCAN state and simply listens to the chosen channel for MEASUREJNTERVAL seconds before returning to the original channel.

During a scan, the MME records "activities" on the chosen channel by means of feature detection (to detect primary devices) or by simply receiving packets (to detect secondary devices). These activities are characterized by several parameters, including the fraction of time that the channel is deemed busy during the scan interval, the average received power and if possible, the type of detected devices (either primary or secondary). These parameters will later be used by RME to identify potential spectral opportunities. Upon completion of a scan, the device returns to the original channel and enters the LISTEN state for LISTEN_INTERVAL seconds before resuming the normal transmission. This extra wait time ensures that the current channel is still available for use by the returning secondary device.

\section{B. Resource Management Entity (RME)}

The RME of each spectral-agile device maintains a spectral opportunity map (SOM), which stores the status of each channel. There are two approaches to updating an SOM: by scanning a channel via MME and by receiving spectralopportunity updates from the RMEs of other devices in the same group. The second approach enables devices to exchange spectral opportunity information so that (1) the work load of discovering spectral opportunities is "distributed" to all devices in the same group, thus avoiding the single-node failure bottleneck, and (2) devices are aware of the hidden primary devices that can only be detected by certain secondary devices. The opportunity update is transmitted upon resumption of transmission after a scan is completed. This information contained in an opportunity update is shown in Figure 7-(a). The "Index" field in the update represents the channel index, the "Duration" field represents the scanning duration (i.e., MEASURE INTERVAL, the "P_/S_utilization" field represents the percentage of the scanning duration when activities from primary/secondary device are detected, and the "P_power" field represents the average detected power of primary devices' transmissions.

Figure 7-(b) shows an example of the SOM maintained by RME. The "Idle" field indicates if a channel is available or not. A value of 1 means that the channel is idle and considered as a spectral opportunity. This field is set to 0 when the latest scan or spectral opportunity update contains a non-zero P_utilization. The "T_Duration field" represents the accumulative amount of time that a spectral-agile group has scanned for that channel. T_Duration is used to compute the average channel utilization of primary and secondary devices (i.e., the "avg_P_util" and "avg_S_util" fields in the SOM). The value of avg_P_util is updated by

$$
\begin{aligned}
& \text { avg_P_util }= \\
& \quad \frac{T \_ \text {Duration } \cdot \text { avg_P_util }+ \text { Duration } \cdot P \_u t i l i z a t i o n}{T \_ \text {Duration }+ \text { Duration }},
\end{aligned}
$$

and so are the values of avg_S_util and avg_P_power. The average channel utilization and average power are useful when more than one channel is available. The RME should always choose the idle channels with the smaller avg_P_util to avoid facing primary devices.

One should note that the information of how long each channel remains available is not included in SOM. Unless the primary devices exhibit predictable usage patterns, such 


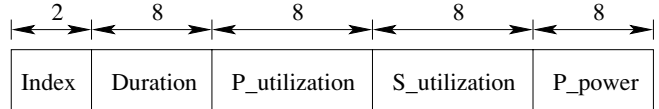

(a) Spectral opportunity update

\begin{tabular}{|c|c|c|c|c|c|}
\hline Index & Idle & T_Duration & avg_P_util & avg_S_util & P_power \\
\hline 0 & 1 & 10 & 0.85 & 0.23 & $-20 \mathrm{db}$ \\
\hline 1 & 0 & 24 & 0.17 & 0 & $-10 \mathrm{db}$ \\
\hline 2 & 0 & $\mathrm{~N} / \mathrm{A}$ & N/A & N/A & N/A \\
\hline & & & & & \\
\hline $\mathrm{N}$ & 1 & N/A & N/A & N/A & N/A \\
\hline
\end{tabular}

(b) Spectral opportunity map

Fig. 7. Spectral opportunity map (SOM).

information is very difficult to obtain or predict since the primary devices may reclaim their channels at any time. Fortunately, spectral-agile devices are required to vacate a channel - within a pre-defied time interval — only upon detection of primary devices' return. Therefore, it is sufficient for spectral-agile devices to know only whether or not a channel is currently available, not how long its availability may last. Of course, such information, if available, can be useful since a spectral-agile device can switch to other idle channels earlier to avoid interfering with primary devices. One should also note that devices in the same spectral-agile group may have different SOMs, because opportunity updates may get corrupted due to transmission errors or missed completely by devices in the SCAN state. As it will be clear in the next subsection, a group-wide, unique SOM is not required in this paper, mainly because of the use of GCE.

\section{Group Coordination Entity (GCE)}

To enable autonomous and cooperative use of spectral opportunities among radio devices of a spectral-agile group or among different spectral-agile groups, we need resourceuse coordination protocols to resolve any potential conflict/contention in utilizing the spectral opportunities. Based on the participants involved in resource-use coordination, we propose two protocols as described below.

1) Intra-group Resource-use Coordination: The most challenging task in realizing spectral-agile communication is to maintain intra-group connectivity in a spectral-agile group. For example, if some devices decide to switch to channel $\mathrm{x}$ while the others decide to switch to channel $\mathrm{y}$, these devices will lose the communication between them. To achieve coordinated channel switching, devices that detect the primary devices will enter the $V A C A N C Y$ state, and search its SOM for any spectral opportunity. If the SOM indicates the availability of certain channels, the device disseminates a notification to inform other devices of an upcoming channel switching. If there is no channel available, the devices remain in the VACANCY state and cancel their next scheduled scan. The reason to cancel the scan is that other devices (in the same spectral-agile group) could have found a spectral opportunity and are about to disseminate a switch notification. If a device leaves the current channel for scanning, it may miss the notification and thus lose the connection with the others.

The device that has prepared a switching notification waits for VACANCY INTERVAL seconds before sending its notification. This additional wait time ensures that devices in the SCAN state will have enough time to complete the scanning, return to the original channel, and receive this notification. This requires that

\section{VACANCYJNTERVAL >}

$$
\text { MEASUREJNTERVAL + LISTEN_INTERVAL. }
$$

Moreover, to avoid transmitting switch notifications at the same time, devices should delay the transmission of their own notifications for extra OFFSET seconds after waiting for VACANCY_INTERVAL seconds. Obviously, each device must have a unique value of OFFSET. To further prevent multiple notifications disseminated within a group, a device with a pending notification must discard such a notification after receiving a switch notification from the others. By doing so, only one unique switch notification is disseminated among devices in the spectral-agile group, thus achieving coordinated channel switching.

Note that it is always possible that a devices may miss a switch notification due to some transmission errors. Therefore, coordinated channel switching cannot always be guaranteed even if other sophisticated retransmission and handshaking mechanisms are applied. Secondary devices may try to establish a group-wide, unified SOM so that, whenever the group needs to vacate a channel, all devices in that group choose the same spectral opportunity by simply checking their SOMs (thus, eliminating the transmission of switching notifications). However, this requires all opportunity updates to be received correctly by all devices, which again requires retransmission and additional handshaking/acknowledgement mechanisms. Since disseminating opportunity updates is more frequent than disseminating channel switching notifications, the proposed approach is more reliable. In any case, devices that lose communication with some devices after switching to a new channel may switch back to the previous communicating channel or a pre-defined channel for re-coordination [18].

2) Inter-group Resource-use Coordination: To make two different spectral-agile groups cooperatively utilize resource opportunities, one needs to ensure that different groups (i) fairly share a single channel when occupying the same channel, and (ii) utilize different spectral opportunities, if there exist multiple opportunities. The first goal can be easily achieved by using the IEEE 802.11 standard-like carrier sense multiple access with collision avoidance (CSMA/CA). To achieve the second goal, we propose a load-balancing algorithm to coordinate the use of multiple opportunities among different spectral-agile groups. When a spectral-agile group detects the presence of any other spectral-agile group, devices in that spectral-agile group immediately check the availability of other opportunities in their own SOMs. If any opportunity other than the currently-utilized one is found, the devices follow the intra-group resource-use coordination procedure so that all devices in one of the groups switch to a new channel.

To prevent all involved spectral-agile groups from renounc- 
ing the currently-utilized opportunity, a delay (unique for each spectral-agile group) is introduced so different groups initiate the above procedures at differen times. If a spectralagile group does renounce the current opportunity (i.e., a channel), those spectral-agile groups that have not vacated yet will cancel their pending intra-group coordination procedure, after perceiving the absence of that leaving spectralagile group. These "staying" spectral-agile groups update the channel status in their SOMs accordingly and repeat the inter-group coordination mechanism, if necessary. This way, we can balance and maximize resource utilization without explicit information exchange between different spectral-agile secondary groups.

\section{The Simulation Results}

The protocols and algorithms described in Section V are implemented in $n s-2$ to evaluate protocol overheads and overall performance. We assume that spectral-agile secondary devices use the IEEE 802.11 standard as their underlying MAClayer protocol. For an illustrative purpose, we also model the primary devices as IEEE 802.11 devices with exclusive access to the designated channels. This is achieved by including a device-type ID in the IEEE 802.11 MAC header. Whenever a secondary device receives a MAC frame with the device-type ID set to PRIMARY, the device suspends any transmission and takes action as explained in Section V. This way, we do not need to implement the details of feature detection, and can focus on the impact of primary devices' usage pattern on the proposed protocols and algorithms.

It should be noted that based on our implementation, secondary devices cease transmission only after a primary device's frame are received. If a primary device attempts to reclaim its channel during a secondary device's transmission, the primary device will defer its transmission according to the IEEE 802.11 standard. This implies that there exists a transient period when secondary and primary devices compete for channel access. However, the IEEE 802.11 MAC uses the CSMA/CA protocol, which in general provides a fair access to contenting devices. If there are a total of $N$ devices, each device has on average an access probability of $\frac{1}{N}$. Therefore, the average time that a tagged device has to wait before it can transmit is no more than $N-1$ frame transmissions. Our simulation results showed that this wait time (experienced by primary devices before they acquire the channel) only lasts for at most tens of milliseconds. Compared to the average ONperiod of primary devices, this transient period is negligible and its impact on primary devices is insignificant.

Since secondary devices are only interested in knowing channel availability, the actual number of primary devices is less important than their spectrum-usage pattern from the secondary devices' perspective. Therefore, we assume that there are only two primary devices on each channel. One of these two devices has an $n s-2$ exponential ON/OFF traffic generator and transmits packets to the other. We also assume that each spectral-agile secondary group is composed of 3 devices. To ensure that each group always has traffic to transmit as assumed in Section III, the devices in the spectralagile group generate traffic using the $n s-2$ constant-bit-rate (CBR) traffic generator. Figure 8 shows the simulation setup for a single spectral-agile group exploiting three channels, each with two primary devices. Here, we assume that devices on the same channel can receive transmission from each other, subject to collisions resulting from the IEEE 802.11 protocol.

Throughout our simulation, we assume that the packet size from all traffic generators is 500 bytes and all devices use 1-Mbps PHY rate for data transmission. As explained in Section V, several parameters are needed to control a spectral-agile device, including MEASURE INTERVAL, LISTEN_INTERVAL, and VACANCYINTERVAL. Based on the packet size and transmission rate chosen above, we let $M E A$ SURE INTERVAL $=20 \mathrm{~ms}$ and LISTENJINTERVAL $=10 \mathrm{~ms}$ so that at least two and one frames can be received during the SCAN and LISTEN state, respectively. We also choose VACANCY_INTERVAL $=40 \mathrm{~ms}$ to satisfy Eq. (19).

We assume that MME of each secondary device randomly selects a channel for scanning upon each scheduled scan. As a result of randomization, it is less likely ${ }^{3}$ that two consecutive updates disseminated within a group contain the similar channel information. We further randomize the time intervals between two consecutive scans scheduled by a device. The average of these time intervals, referred to as a device's scan period, is denoted as SCAN_PERIOD and will be a control parameter in our simulation. The advantage of this randomized scheme over using a fixed scan period is that in the latter case, a device may always miss another device's opportunity updates if one's scheduled scan starts right after the end of another's. We will explore different scan periods later and evaluate its impact on secondary devices' performance. Obviously, a smaller SCAN_PERIOD provides more accurate channel information but incurs more overhead and interrupts data transmission more frequently.

Finally, the opportunity updates and switching notifications sent by RME and GCE are coded as new IEEE 802.11 command frames. These command frames are sent with the highest priority. Moreover, switching-notification frames are sent even when the primary devices' has reclaimed the channel. This simplifies our in-band signaling for coordinated switching without using a common control channel. Doing do will interfere with primary devices' transmission but it only takes one-frame transmission time from the primary devices. Throughout our simulation, we only consider the case of $N=3$ as only three non-overlapping channels are available in the current IEEE $802.11 \mathrm{~b}$ wireless LAN. Of course, our implementation works for other scenarios with arbitrary numbers of channels.

\section{A. Throughput Improvement for a Single Spectral-agile Group}

We choose SCAN_PERIOD $=0.5$ second, $T_{\text {on }}=10 *$ channel load seconds, and $T_{\text {off }}=10 *(1-$ channel load $)$ seconds in this simulation. Figure 9 shows the improvements of a spectral-agile group's throughput as compared to that without spectrum agility. Here, we compare the throughput (i.e., the number of packets transmitted), instead of the channel time each group acquires, as it is easier to obtain the throughput in $n s-2$. We consider both homogeneous and

${ }^{3}$ Compared to the case when each MME uses a fixed list of ordered channels and scan them in a round-robin fashion 


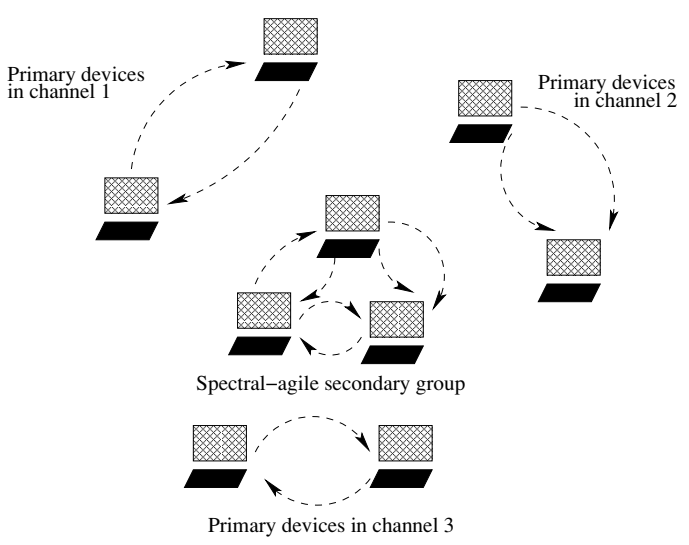

Fig. 8. Simulation setup for a single spectral-agile group exploiting three channels: $N=3$ and $M=1$.

heterogeneous loads, and the simulation results are compared with the analytical results (in solid lines). The improvement obtained from the simulation is shown to be very close to the analytical upper bound in the cases when the average channel load ranges between 0.3 and 0.6. Within this region, the improvement ranges between 40 and $80 \%$ for homogeneous loads, and ranges between 50 and $90 \%$ for heterogeneous loads. Considering the control overhead incurred by spectrum agility, the results confirm the effectiveness of our implementation.

One interesting observation is that the improvement is much less than the analytical results as the channel load increases. The improvement even becomes negative (e.g., $22 \%$ ), meaning that it is even worse than without using spectrum agility, when the primary devices are extremely active. The main reason for this is that when the channel is heavily-loaded, a spectral-agile group is left with very few spectral opportunities. As a result, the scanning, frequent switching and listening simply interrupt the secondary device's normal transmission without providing any additional spectral opportunities. Under this heavy load scenario, staying in a fixed channel would be the best strategy.

Figure 9 also confirms that when the loads of the channels are diverse (i.e., heterogeneous loads), a spectral-agile group achieves better performance as shown in Section III. One can make an extra 10 to $15 \%$ improvement since our implementation enables a secondary group to seek the leastutilized channels and stay there most of time, thus making best use of spectral opportunities.

\section{B. Throughput Improvement of Multiple Spectral-agile groups}

The previous simulation shows that the throughput of a single spectral-agile group increased by up to $90 \%$. We now use $N=3$ and $M=2$ to investigate how different spectralagile secondary groups interact with each other when seeking and utilizing spectral opportunities. The simulation is set up as shown in Figure 10. For an illustrative purpose, we only simulate the case of homogeneous channel loads and set SCAN_PERIOD $=0.5$ second. In order to make these two spectral-agile groups share the spectral opportunities, instead of letting them compete for these opportunities, we assign different priorities to each spectral-agile group. The priority is used by a spectral-agile group to determine the value of

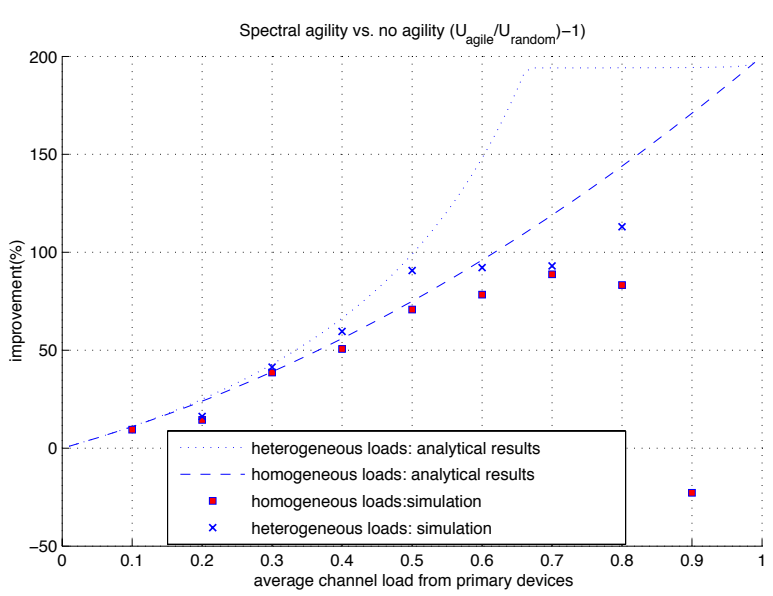

Fig. 9. Throughout improvement percentage as compared to the case of no agility with random channel selection for $N=3$ and $M=1$. *The substantial discrepancy between the numerical analysis and simulation results from that our analytical model does not consider any protocol overhead. These overheads easily consume the minuscule channel access time (as shown in Figure 4) gained by spectrum agility when the load is close to 1 .

delay in the inter-group resource-use cooperation algorithm (in Section V). It should be noted that the lower-priority group is not discriminated in terms of using spectral opportunities. Our simulation results show that these two spectral-agile groups always achieve almost the same throughput.

Figure 11 shows the improvement of spectral-agile groups' average throughput, as compared to the case of using no agility with off-line channel allocation. In general, the improvements are very close to the analytical results (within a $13 \%$ margin). In some cases, the simulation shows a larger improvement percentage than the analytical value. This is because after detecting the presence of primary devices, our implementation also requires non-agile secondary groups to suspend all transmissions for VACANCY_INTERVAL seconds before attempting to use that channel again. For a spectral-agile group, it is less likely to encounter a busy channel due to spectrum agility. Therefore, the time wasted during the suspension in a nonagile group is relatively larger than a spectral-agile group. As a result, the improvement percentage is higher than the analytical result, especially when the channel is moderatelyloaded.

One can also observe that using spectrum agility results in poorer performance $(-9 \%)$ than without using agility, when the channels are heavily-loaded. Again, it does not make any sense to use spectral agility in those heavily-loaded channels as virtually no opportunity exists in those channels. In this case, the overhead easily offsets any improvement made by spectrum agility as in the case of a single spectral-agile group.

So Far, the simulation results demonstrate a very important advantage of using spectrum agility: by using spectrum agility, we can achieve a higher throughput (more than $30 \%$ in many cases, as compared to using no agility with off-line channel allocation, let alone an even higher improvement as compared to using random channel selection) without any offline planning on spectral resource allocation. That is, using spectrum agility easily achieves the automated frequency use coordination as we mentioned in Section I and results in a much higher spectral utilization. 


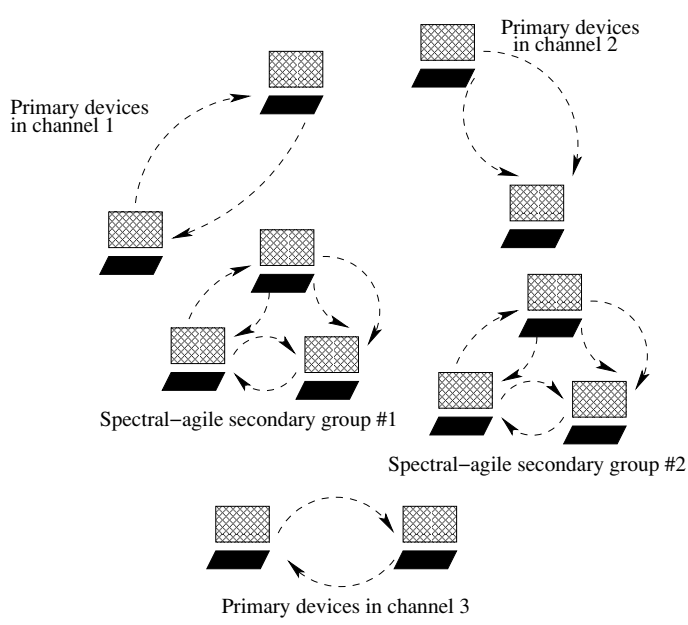

Fig. 10. Simulation setup for multiple spectral-agile groups: $N=3$ and $M=2$.

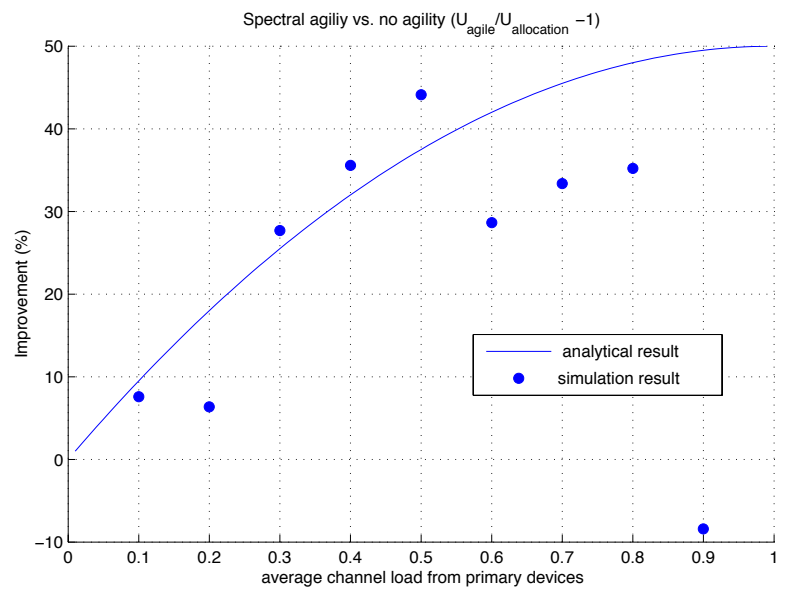

Fig. 11. Throughout improvement percentage as compared to the case of no agility with off-line channel allocation: $N=3$ and $M=2$. *The substantial discrepancy between the numerical analysis and simulation exists because our analytical model does not consider any protocol overhead. However, these overheads easily consume the minuscule channel access time (as shown in Figure 4) gained by spectrum agility when the load is close to 1 .

\section{Improvements vs. SCAN_PERIOD}

We now investigate the effects of SCAN_PERIOD on the improvement of a spectral-agile group's throughput. We consider three different loads generated by the primary devices (0.2, 0.5 and 0.8$)$, and let $T_{o n}=10 *$ channel load seconds and $T_{\text {off }}=10 *(1-$ channel load $)$ seconds. Figure 12 shows that for a fixed channel load, the improvement decreases with the increase of SCAN_PERIOD. This is because the channel availability can be determined with a higher probability by increasing the frequency of channel scanning. Of course, how small a SCAN_PERIOD should be, depends on the channel load generated by the primary devices. As one can observe from Figure 12, doubling the SCAN_PERIOD from 0.5 second to 1 second does not degrade the secondary group's throughput at all, when the channel loads are 0.2 and 0.5 . However, in the case of heavy load (e.g., 0.8), the throughput decreases sharply with the increase of scan periods. This shows that the impact of scan periods on secondary devices' performance depends on the channel loads. A secondary device should choose the optimal scan period according to the channel loads generated by the primary devices.

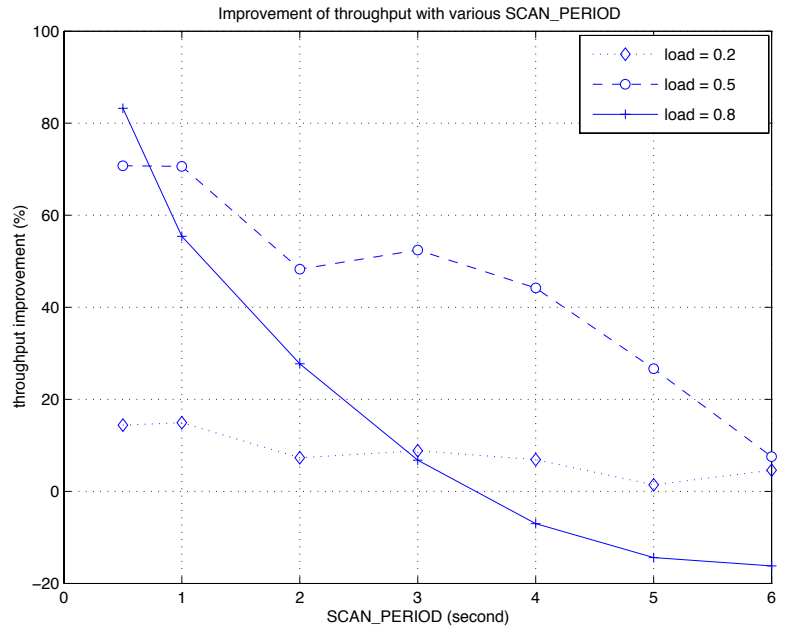

Fig. 12. Impacts of SCAN_PERIOD on the spectral-agile group's throughput improvement.

\section{Improvements for Different Primary Devices' Usage Pat- terns}

As discussed above, the throughput improvement of a spectral-agile group is determined by SCAN_PERIOD and the average loads generated by the primary devices. However, the channel load only does not fully characterize primary device's spectrum usage. One can expect that a smaller SCAN_PERIOD is needed for a more dynamic channel than a static channel, even though both have the same average load. We investigate this issue as follows. We choose 3 different values of SCAN_PERIOD. For each SCAN_PERIOD value, we change the $T_{o n}$ and $T_{\text {off }}$ while keeping the channel load $\left(=\frac{T_{o n}}{T_{o n}+T_{o f f}}=0.5\right)$ unchanged. The total number of packets transmitted (by the spectral-agile group) within a 1000-second interval is plotted in Figure 13.

For any given value of SCAN_PERIOD, the number of transmitted packets generally increases with the decrease of dynamics in primary devices' usage pattern (i.e., increase of $T_{o n}$ and $\left.T_{o f f}\right)$. This is because spectral-agile devices need not switch between channels very frequently when the channel is not highly dynamic, even though the channel load is fixed at 0.5 for all cases. One should also observe a slight decrease in improvement after the average ON- and OFF-periods exceeds 4.0 seconds for the case of $S C A N_{-} P E R I O D=0.5$. This is because scanning a less-dynamic channel too frequent does not provide more opportunities bur only incur more overheads. However, as compared to using a larger SCAN_PERIOD, using a smaller SCAN_PERIOD always achieves much better performance even though the scanning overhead increases linearly with the scanning frequency. This is because the overhead incurred by scanning is relatively small in our implementation (only MEASURE INTERVAL+LISTEN INTERVAL $=0.03$ second for every SCAN_PERIOD $=0.5$ second).

We can conclude that the improvement via spectral-agile devices is primarily determined by the value of SCAN_PERIOD. A spectral-agile device should choose a SCAN_PERIOD based on channel loads and more importantly, the duration of ON- and OFF-periods, of each channel. If the channels switch between ON- and OFF-periods very often, a smaller 


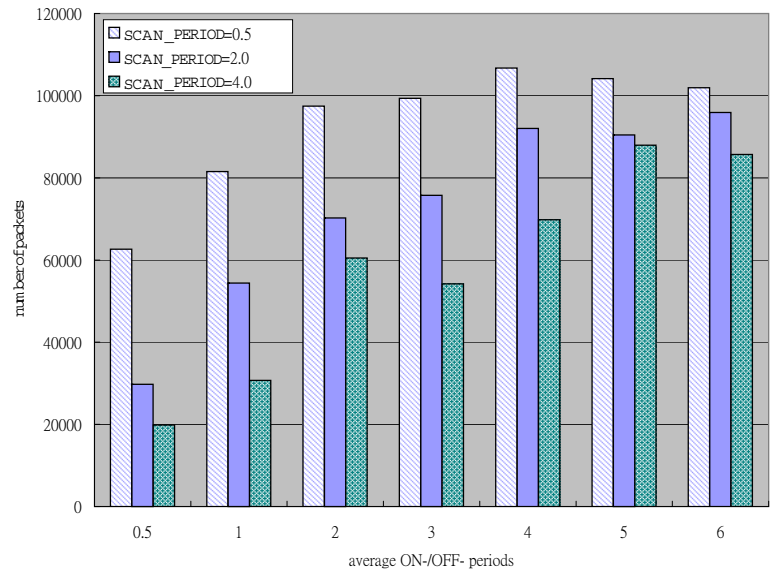

Fig. 13. Effects of SCAN_PERIOD vs. Effects of average $O N$-/OFF-period on the spectral-agile group's throughput.

SCAN_PERIOD is required. However, a large SCAN_PERIOD may suffice to discover opportunities without degrading performance too much. Our results suggest that choosing the SCAN_PERIOD as one fourth to one eighth of the primary devices' ON- or OFF- periods makes a pretty good improvement in the simulated scenarios.

\section{CONCLUSIONS AND Future WORK}

In this paper, we have investigated the issue of using spectrum agility to improve both spectrum utilization and secondary device's performance. We established a simple mathematical model and provide performance benchmarks for general spectral-agile communication. The results based on this model have shown that the channel utilization of a spectral-agile device is improved by 35 to $200 \%$ when compared to the cases of no agility, depending on the number of channels, their average channel load, the number of spectralagile devices.

In order to realize spectral-agile communication, we developed three basic building blocks for spectral-agile secondary devices, including MME, RME and GCE. The protocols and algorithms in each building block have been implemented in the IEEE 802.11 wireless LAN in the $n s-2$. The simulation results show that the improvement of a secondary device's throughput: (1) can be up to $90 \%$ by using spectrum agility, (2) is close to the performance benchmark predicted by our analytical model, and (3) is achieved in a distributed and automated manner with little overhead. We have shown that our protocol easily outperforms the static, off-line channel allocation. We are currently examining spectral-agile devices which use multiple spectral opportunities simultaneously, and studying the MAC-layer protocol to further improve secondary device's performance.

\section{REFERENCES}

[1] "Facilitating Opportunities for Flexible, Efficient, and Reliable Spectrum Use Employing Cognitive Radio Technologies," the FCC Notice of Proposed Rulemaking and Order - ET Docket No. 03-108.

[2] http://www.darpa.mil/ato/programs/XG/rfc_vision.pdf - Vision RFC.

[3] C. Bergstrom, S. Chuprun and D. Torrieri, "Adaptive spectrum exploitation using emerging software defined radios", IEEE Radio and Wireless Conference 1999, pp.113-116.
[4] P. K. Lee, "Joint frequency hopping and adaptive spectrum exploitation", IEEE MILCOM 2001, Vol.1, pp.566-570.

[5] C. Cordiero, K. Challapali, D. Birru and S. Shankar,'IEEE 802.22: The first world wide standard for Cognitive Radio", IEEE DySPAN Conference 2005, USA.

[6] J. Mitola, "The software radio architecture", IEEE Communications, Vol.33, No.5, 1995, pp.26-38.

[7] "White paper on regulatory aspects of software defined radio", $S D R$ forum document number SDRF-00-R-0050-v0.0.

[8] http://www.darpa.mil/ato/programs/XG/rfc_af.pdf - Architecture RFC.

[9] J.B. Punt, D. Sparreboom, F. Brouwer and R. Prasad, "Mathematical analysis of dynamic channel selection in indoor mobile wireless communication systemse", IEEE Transactions onVehicular Technology, Vol.47, No.4, Nov. 1998, pp.1302-1313.

[10] "IEEE 802.11h standard (Amendment to IEEE 802.11 Standard, 1999 Edition)", 2003.

[11] J. Khun-Jush, G. Malmgren, P. Schramm and J. Torsner, "Overview and performance of HIPERLAN type 2-a standard for broadband wireless communications", IEEE VTC 2000-Spring, Tokyo, Vol.1, pp.112 - 117.

[12] "Additional spectrum for unlicensed devices below $900 \mathrm{MHz}$ and in the $3 \mathrm{GHz}$ band", FCC ET Docket No. 02-380.

[13] "Future trends in defence antenna technology", http://www.bcba15324.pwp.blueyonder.co.uk/consulting/Bibliography/ Paper-16.pdf.

[14] D. Bertsekas and R. Gallager, "Data Networks", Prentice-Hall, New York, 1992.

[15] A. Sahai, D. Cabric, N. Hoven, R. Tandra, S.M. Mishra, and R. Brodersen,"Spectrum sensing: fundamental limits and practical challenges," Tutorial presented at the IEEE DYSPAN 2005 Conference.

[16] R. Kolodziejski and J. Betz, "Detection of Weak Random Signals in IDD Non-Gaussian Noise," IEEE Transaction on Communication, Vol. 48, No. 2, February 2000.

[17] C.W. Gardiner, "Handbook of Stochastic Methods", Second Edition, Springer.

[18] X. Jing and D. Raychaudhuri, "A Spectrum Etiquette Protocol for Efficient Coordination of Radio Devices in Unlicensed Bands", Proceedings of IEEE PIMRC'03, Sep. 2003

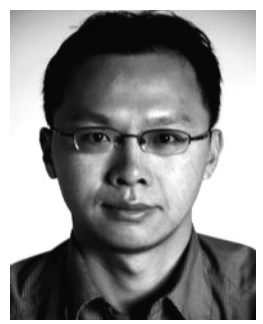

Chun-Ting Chou (M '05/ACM '05) is currently a senior member research staff in Philips Research USA, Briarcliff Manor, New York. He received both the B.S. and M.S. degrees in Electrical Engineering from National Taiwan University, Taipei, Taiwan in 1995 and 1997, respectively. He received the $\mathrm{Ph} . \mathrm{D}$. degree in Electrical Engineering and Computer Science from the University of Michigan, Ann Arbor, Michigan in 2004. He is actively involved in standardization of WiMedia MAC protocol, and IEEE $802.15 .3 \mathrm{c}$ and Ecma International TG20 for 60-Ghz short-range wireless transmission. His current research interests include design and analysis of protocols in wireless and mobile networks, Ultra-Wide Band (UWB) wireless personal area networks, multi-gigabyte short-range transmission, and spectrum agile and cooperative communication.

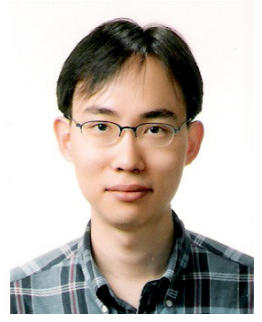

Hyoil Kim is currently a Ph.D. candidate in the department of Electrical Engineering and Computer Science, the University of Michigan. He received his B.S. degree from Seoul National University in 1999, and M.S. degree from the University of Michigan in 2005, respectively. Since 2004, he has been a research assistant in the Real-Time Computing Laboratory in EECS Department, the University of Michigan. His research interests include Dynamic Spectrum Access, Cognitive Radio Networks and wireless spectrum resource allocation/management. 


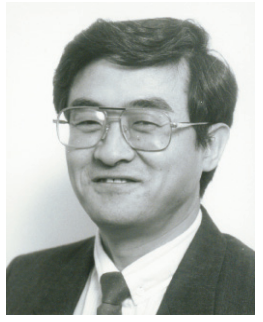

Kang G. Shin (F '92) is the Kevin and Nancy O'Connor Professor of Computer Science and Founding Director of the Real-Time Computing Laboratory in the Department of Electrical Engineering and Computer Science, The University of Michigan, Ann Arbor, Michigan. His current research focuses on QoS-sensitive networking and computing as well as on embedded real-time OS, middleware and applications, all with emphasis on timeliness and dependability. He has supervised the completion of $56 \mathrm{PhD}$ theses, and authored/coauthored around 630 technical papers and numerous book chapters in the areas of distributed real-time computing and control, computer networking, fault-tolerant computing, and intelligent manufacturing. He has co-authored (jointly with C. M. Krishna) a textbook "Real-Time Systems," McGraw Hill, 1997.

$\mathrm{He}$ has received a number of best paper awards, including the IEEE Communications Society William R. Bennett Prize Paper Award in 2003, the Best Paper Award from the IWQoS'03 in 2003, and an Outstanding IEEE Transactions of Automatic Control Paper Award in 1987. He has also coauthored papers with his students which received the Best Student Paper Awards from the 1996 IEEE Real-Time Technology and Application Symposium, and the 2000 UNSENIX Technical Conference. He has also received several institutional awards, including the Research Excellence Award in 1989, Outstanding Achievement Award in 1999, Service Excellence Award in 2000, Distinguished Faculty Achievement Award in 2001, and Stephen Attwood Award in 2004 from The University of Michigan; a Distinguished Alumni Award of the College of Engineering, Seoul National University in 2002; and 2003 IEEE RTC Technical Achievement Award.

He received the B.S. degree in Electronics Engineering from Seoul National University, Seoul, Korea in 1970, and both the M.S. and Ph.D degrees in Electrical Engineering from Cornell University, Ithaca, New York in 1976 and 1978, respectively. From 1978 to 1982 he was on the faculty of Rensselaer Polytechnic Institute, Troy, New York. He has held visiting positions at the U.S. Airforce Flight Dynamics Laboratory, AT\&T Bell Laboratories, Computer Science Division within the Department of Electrical Engineering and Computer Science at UC Berkeley, and International Computer Science Institute, Berkeley, CA, IBM T. J. Watson Research Center, Software Engineering Institute at Carnegie Mellon University, and HP Research Laboratories. He also chaired the Computer Science and Engineering Division, EECS Department, The University of Michigan for three years beginning January 1991.

He is Fellow of IEEE and ACM, and member of the Korean Academy of Engineering, is serving as the General Chair for the 3rd ACM/USENIX International Conference on Mobile Systems, Applications, and Services (MobiSys'05), was the General Chair of the 2000 IEEE Real-Time Technology and Applications Symposium, the Program Chair of the 1986 IEEE Real-Time Systems Symposium (RTSS), the General Chair of the 1987 RTSS, the Guest Editor of the 1987 August special issue of IEEE Transactions on Computers on Real-Time Systems, a Program Co-Chair for the 1992 International Conference on Parallel Processing, and served numerous technical program committees. He also chaired the IEEE Technical Committee on Real-Time Systems during 1991-93, was a Distinguished Visitor of the Computer Society of the IEEE, an Editor of IEEE Trans. on Parallel and Distributed Computing, and an Area Editor of International Journal of Time-Critical Computing Systems, Computer Networks, and ACM Transactions on Embedded Systems.

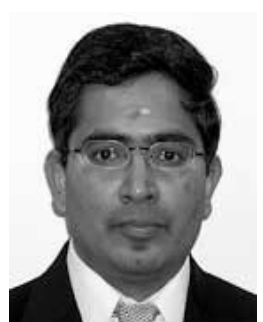

Sai Shankar N (SM '05/ACM '05) received his $\mathrm{PhD}$ degree from the department of Electrical Communication Engineering from Indian Institute of Science, Bangalore, India for his work in the area of performance modeling of ATM networks. In 1998, He was awarded the German Fellowship, DAAD, in the department of mathematics, University of Kaiserslautern, Gernany to work on queueing approaches in manufacturing. In 1999, he joined Philips Research, Eindhoven, the Netherlands, where he served as Research Scientist in the department of New Media Systems and Applications working on various problems involving Hybrid, Fiber, Co-axial Cable (IEEE 802.14) Networks and IP protocols and provided efficient algorithms to improve protocol efficiency. In the year 2001 he joined Philips Research USA, Briarcliff Manor, NY and worked in the area of Wireless LANs/Ultra Wide Band (UWB), Cognitive Radios and Cooperative Communications. He was a key member in shaping the QoS related issues in IEEE 802.11e standardization and was one of the prime authors and inventors of the WiMEDIA UWB MAC protocol for which he was nominated as one of the five finalists in the Innovator of the year category by EE Times in year 2004. He has chaired numerical conferences and technical sessions and has delivered more than ten tutorials in leading international conferences of which two will appear in IEEE COMSOC webpage. He is also the Senior Member of the IEEE and has authored more than 50 conference and journal papers and holds more than 35 patents. Currently Sai Shankar is with Corporate R\&D Systems Engineering department at Qualcomm Inc. working on issues related to $4 \mathrm{G}$ systems, UWB, cooperative communications and cognitive radios. 